\title{
Caracterização dos casos de tuberculose notificados em um município prioritário do Brasil, de 2011-2015
}

\author{
Characterization of tuberculosis cases reported in a priority municipality of Brazil, from \\ 2011-2015
}

Caracterización de los casos de tuberculosis notificados en un municipio prioritario de Brasil, de 2011-2015

Susiele Maria de Arruda Lima ${ }^{1 *}$, Elisama Melquiades de Melo e Silva ${ }^{1}$, Maria Jose de Lima ${ }^{1}$, Angélica de Melo Jucá2.

\section{RESUMO}

Objetivo: Analisar epidemiologicamente o número dos casos notificados de tuberculose no município prioritário do Brasil, Recife nos anos de 2011 a 2015. Método: Trata-se de um estudo epidemiológico, retrospectivo, descritivo e analítico de uma série histórica no período de 2011 a 2015 no município do Recife, utilizou-se a base de dados do DATASUS/SINAN/SMS, de casos de tuberculose. Após a coleta foram tabulados os dados e realizado a análise descritiva simples utilizando o Excel. Resultados: Constatou-se que no período de 2011 a 2015, foram realizadas 12.973 notificações de caso de tuberculose no Recife, durante os 5 anos analisados, revelou que $68,11 \%$ dos portadores da doença eram homens, na faixa etária de $20-49$ anos $45,12 \%, 62,08 \%$ ocorrem em indivíduos não institucionalizados, a admissão de $75,48 \%$ ocorreram por caso novo, em $61,53 \%$ dos casos foi realizada a baciloscopia, a forma pulmonar prevaleceu com $80,84 \%$, A sorologia para o HIV foi realizada em $57,76 \%$ dos casos. Em relação a situação de encerramento dos casos de TB, a maioria evoluiu para a cura $46,35 \%$. Conclusão: Ao caracterizar o perfil epidemiológico dos casos notificados por tuberculose no Recife, este estudo contribui com ações de prevenção e controle da doença no município.

Palavras-chave: Tuberculose, Epidemiologia, Notificação de doenças.

\begin{abstract}
Objective: This paper aims to analyze epidemiologically the number of reported cases of tuberculosis in the priority municipality of Brazil, Recife in the years 2011 to 2015. Method: This is an epidemiological, retrospective, descriptive and analytical study of a historical series in the period from 2011 to 2015 in the municipality of Recife. The DATASUS / SINAN / SMS database of tuberculosis cases will be used. After the data collection, the data were tabulated. Simple descriptive analysis will be performed using the Excel spreadsheet software. Results: It was verified that in the period from 2011 to 2015, 12,973 cases of tuberculosis were reported in Recife, during the 5 years analyzed, revealed that $68.11 \%$ of the patients with the disease were men, in the age group of $20-49$ years $45,12 \%, 62.08 \%$ occur in non-institutionalized individuals, $75.48 \%$ admission occurred by NOVO case, in $61.53 \%$ of the cases smear was performed, pulmonary form prevailed with $80.84 \%$, serology for HIV was performed in $57.76 \%$ of the cases. In relation to the situation of closure of TB cases, the majority evolved to cure $46.35 \%$. Conclusion: In characterizing the epidemiological profile of tuberculosis cases reported in Recife, this study will contribute to prevention and control of the disease in the municipality.
\end{abstract}

Keywords: Tuberculosis; Epidemiology; Notification of illness.

${ }_{1}$ Universidade Federal de Pernambuco (UFPE), Recife-Pernambuco. *E-mail: susielelima@yahoo.com.br

2 Secretaria de Saúde de Pernambuco (SES-PE). 


\section{RESUMEN}

Objetivo: Analizar epidemiológicamente el número de casos notificados de tuberculosis en el municipio prioritario de Brasil, Recife en los años 2011 a 2015. Método: Se trata de un estudio epidemiológico, retrospectivo, descriptivo y analítico de una serie histórica en el período de 2011 a 2015 en el municipio de Recife, se utilizó la base de datos de DATASUS / SINAN / SMS, de casos de tuberculosis después recolección fueron tabulados los datos y realizado el análisis descriptivo simple utilizando Excel. Resultados: Se constató que en el período de 2011 a 2015 se realizaron 12.973 notificaciones de casos de tuberculosis en Recife, durante los 5 años analizados, reveló que el $68,11 \%$ de los portadores de la enfermedad eran hombres, en el grupo de edad de 20-49 En la mayoría de los casos, se observó un aumento de la mortalidad por cáncer de próstata en los últimos años, La serología para el VIH se realizó en el $57,76 \%$ de los casos. En cuanto a la situación de cierre de los casos de TB, la mayoría evolucionó a la cura $46,35 \%$. Conclusión: Al caracterizar el perfil epidemiológico de los casos notificados por tuberculosis en Recife, este estudio contribuye con acciones de prevención y control de la enfermedad en el municipio.

Palabras clave: Tuberculosis; epidemiología; Notificación de enfermedades.

\section{INTRODUÇÃO}

A Tuberculose (TB) permanece sendo um grave problema mundial de saúde. Segundo a Organização Mundial de Saúde (OMS) em 2015 cerca de 10,4 milhões de pessoas tiveram tuberculose e mais de 1 milhão morreram por conta da enfermidade. A OMS definiu para o período de 2016 a 2020 listas de países prioritários para a abordagem da tuberculose, o Brasil se encontra em duas listas, ocupando a $20^{a}$ posição quanto à carga da doença e a 19aㅡ no que se refere à confecção TB-HIV (BRASIL, 2017).

Diante desse panorama o Ministério da Saúde (MS) construiu o Plano Nacional pelo Fim da Tuberculose e tem como metas reduzir, até o ano de 2035, a incidência para 10/100 mil habitantes e a taxa de mortalidade para menos de 1 óbito para 100 mil habitantes, em relação a 2015 (BRASIL, 2017).

A situação epidemiológica da tuberculose no Brasil é alarmante, em 2015 foram registrados 4.543 óbitos, o estado de Pernambuco apresentou o segundo maior risco para o óbito por tuberculose 4,5/100 mil habitantes e no que concerne as capitais, o município do Recife apresentou o maior risco de morte 7,7/100 mil habitantes (BRASIL, 2015).

A TB é uma doença infecciosa e crônica, seu agente etiológico predominante é o bacilo aeróbico Mycobacterium tuberculosis, também denominado como bacilo de Koch (BK), mas pode ser causada por qualquer uma das sete espécies que integram o complexo Mycobacterium tuberculosis (BOMBARDA S, et al., 2014). Essa espécie trata-se de uma bactéria aeróbica, ácido-resistente que se prolifera lentamente, é fotossensível e sensível ao calor (ARAÚJO SRL, et al., 2015; FREITAS VMTM, et al., 2016).

O bacilo afeta principalmente o ápice dos pulmões e alvéolos, uma vez que a oferta de oxigênio é maior para sua multiplicação, porém pode acometer qualquer órgão do corpo humano como, linfonodos, ossos, rins e meninges (OLIVEIRA GM, PETRONI TF, 2017; PEREIRA VLT, et al., 2017). Para a saúde pública a tuberculose pulmonar é a forma mais alarmante, pois além de ser a mais frequente é a principal responsável pela transmissão da doença (FREITAS VMTM, et al., 2016).

A transmissão da TB ocorre por via aérea. O indivíduo doente dissemina o bacilo para o sujeito sadio através da respiração, fala, espirro, tosse, risos ou qualquer outra forma de relação pessoal que libere gotículas de saliva contaminadas (ARAÚJO SRL, et al., 2015; FREITAS VMTM, et al., 2016; PEREIRA VLT, et al., 2017).

Os episódios de tuberculose, sejam individuais ou coletivos, trazem uma forte ligação com os aspectos socioeconômicos em que se encontram as pessoas acometidas. Dentre os aspectos que estão associados à infecção nota-se condições precárias de vida, falta de higiene, má qualidade de alimentação e moradias pouco ventiladas. Os privados de liberdade, moradores de rua, os acometidos por HIV/AIDS e povos indígenas constituem grupos de riscos devido às condições de saúde e de vida a que estão submetidos (OLIVEIRA GM, PETRONI TF, 2017; LIMA SS, et al., 2017). 
A aglomeração de pessoas associado à imunidade baixa são as principais formas de contágio e uma vez o indivíduo sendo infectado, a manifestação da TB pode ocorrer em qualquer momento da vida (FREITAS VMTM, et al., 2016; OLIVEIRA GM e PETRONI TF, 2017).

Os pacientes infectados pelo bacilo apresentam febre vespertina, tosse, rouquidão, sudorese noturna, fadiga, falta de apetite e perda ponderal. A tosse pode ser acompanhada de expectoração mucopurulenta ou não, como também pode ocorrer hemoptise (ARAÚJO SRL, et al., 2015; CHAVES J, et al., 2017; OLIVEIRA GM e PETRONI TF, 2017).

A presença destes sinais e sintomas contribuem para o diagnóstico clínico da doença. O diagnóstico laboratorial se dá pela realização de exames de cultura de escarro, radiografia de tórax, baciloscopia direta do escarro e o teste tuberculínico (PPD) (ARAÚJO SRL, et al., 2015; CHAVES J, et al., 2017; PEREIRA VLT, et al., 2017). O tratamento é medicamentoso, dura em média de 6 meses a 1 ano, é gratuito e pode ser feito em regime ambulatorial. Trata-se de uma doença de fácil diagnóstico, curável e evitável (ALVES RH, et al., 2014; FREITAS VMTM, et al., 2016).

Os desafios para as políticas de controle da tuberculose consistem em enfrentar problemas em relação a resistência medicamentosa, recidiva da doença, tratamento prolongado, falta de adesão ao tratamento e a associação da TB com o HIV (OLIVEIRA JHS, et al., 2015).

A avaliação da atual situação epidemiológica da tuberculose em Recife permitirá que as autoridades de saúde possam traçar estratégias para o combate da doença no município do Recife (PE).

Nesse sentido, este trabalho teve como objetivo analisar a tuberculose em Recife (PE), verificando a sua prevalência, perfil clinico epidemiológico dos pacientes, bem como a existência de agravos associados (HIV) e a situação dos casos notificados.

\section{MÉTODOS}

Trata-se de um estudo epidemiológico, retrospectivo, descritivo e analítico de uma série histórica no período de 2011 a 2015 no município do Recife-Pernambuco. A população alvo foi de todos os casos de tuberculose notificados no SINAN/SMS, no município do Recife, PE no período de 2011 a 2015.

Foram incluídos no estudo todos os casos notificados e com diagnóstico confirmado de tuberculose no município do Recife e excluídos da análise todos os casos que, apesar de notificados, não apresentaram confirmação diagnóstica ou que continham inconsistências.

A fonte de dados foi secundária, os registros foram coletados a partir da base de dados do DATASUS/SINAN/SMS, de casos de tuberculose. Os dados foram obtidos através de relatórios operacionais no TABWINTB instalado no mesmo sistema. Dispensou-se a submissão ao Comitê de Ética em Pesquisa com Seres Humanos (CEP), pois este estudo utilizou dados secundários disponíveis em base de dados governamental de domínio público.

Foram coletadas do SINAN informações relacionadas à descrição sociodemográfica como sexo, idade, escolaridade e a aspectos da doença como localização, doenças associadas, tipo de entrada, exames realizados e situação de encerramentos. Após a coleta dos dados foi realizada a análise descritiva simples utilizando o software de planilha eletrônica Excel. Os achados mais significantes foram apresentados por tabelas e gráficos. A pesquisa não se constituiu de riscos visto que envolveu a utilização de dados secundários em alguns bancos disponíveis ao público, onde os indivíduos não podem ser identificados.

\section{RESULTADOS}

Neste estudo foi observado que no período de 2011 a 2015, foram realizadas 12.973 notificações de caso de tuberculose no município do Recife, com uma média de 2.594,4 notificações por ano. No ano de 2013 ocorreu a maior frequência de notificações $(n=2.723)$, seguido de $2012(n=2628)$ e a menor frequência ocorreu no ano de 2015 ( $n=2476)$ (Tabela 1). 
A distribuição dos casos por gêneros, durante os 5 anos analisados, revelou que $8.835(68,11 \%)$ dos portadores da doença eram homens e houve em todos os anos uma prevalência do sexo masculino. A relação de incidência entre os sexos foi de 2,1:1 (Tabela 1). Quanto as formas clínicas da tuberculose, a forma pulmonar prevaleceu com 10.487 casos $(80,84 \%)$, seguida da forma extrapulmonar com $1.821(14,04 \%)$ (Tabela 1).

Tabela 1 - Caracterização dos casos de tuberculose no município de Recife/PE segundo Sexo, Forma Clínica e Tipo de Admissão no período de $2011-2015$.

\begin{tabular}{llllllll}
\hline & $\mathbf{2 0 1 1}$ & $\mathbf{2 0 1 2}$ & $\mathbf{2 0 1 3}$ & $\mathbf{2 0 1 4}$ & $\mathbf{2 0 1 5}$ & Total & Total \% \\
\hline Sexo & & & & & & & \\
\hline Masculino & 1704 & 1790 & 1866 & 1805 & 1670 & 8835 & 68,10 \\
Feminino & 884 & 838 & 856 & 752 & 806 & 4136 & 31,88 \\
Ignorado & 0 & 0 & 1 & 1 & 0 & 2 & 0,02 \\
Total & 2588 & 2628 & 2723 & 2558 & 2476 & 12973 & 100,00 \\
\hline Forma Clínica & & & & & & & \\
\hline Pulmonar & 2106 & 2083 & 2198 & 2065 & 2035 & 10487 & 80,84 \\
Extrapulmonar & 362 & 398 & 365 & 370 & 326 & 1821 & 14,04 \\
Pulmonar e Extrapulmonar & 120 & 146 & 160 & 122 & 113 & 661 & 5,10 \\
Ignorado/Branco & 0 & 1 & 0 & 1 & 2 & 4 & 0,03 \\
Total & 2588 & 2628 & 2723 & 2558 & 2476 & 12973 & 100,00 \\
\hline Tipo de Admissão & & & & & & & \\
\hline Novo & 1983 & 2068 & 2076 & 1935 & 1730 & 9792 & 75,48 \\
Reingresso após abandono & 259 & 275 & 351 & 363 & 343 & 1591 & 12,26 \\
Recidiva & 230 & 190 & 187 & 171 & 161 & 939 & 7,24 \\
Transferência & 91 & 71 & 74 & 71 & 124 & 431 & 3,32 \\
Pós Óbito & 0 & 0 & 3 & 5 & 102 & 110 & 0,85 \\
Não sabe & 25 & 24 & 32 & 13 & 16 & 110 & 0,85 \\
\hline Total & 2588 & 2628 & 2723 & 2558 & 2476 & 12973 & 100,00 \\
\hline
\end{tabular}

Fonte: SINAN 2011 - 2015, DATASUS.

Figura 1 - Casos notificados de tuberculose no município de Recife/PE segundo faixa etária no período de 2011-2015.

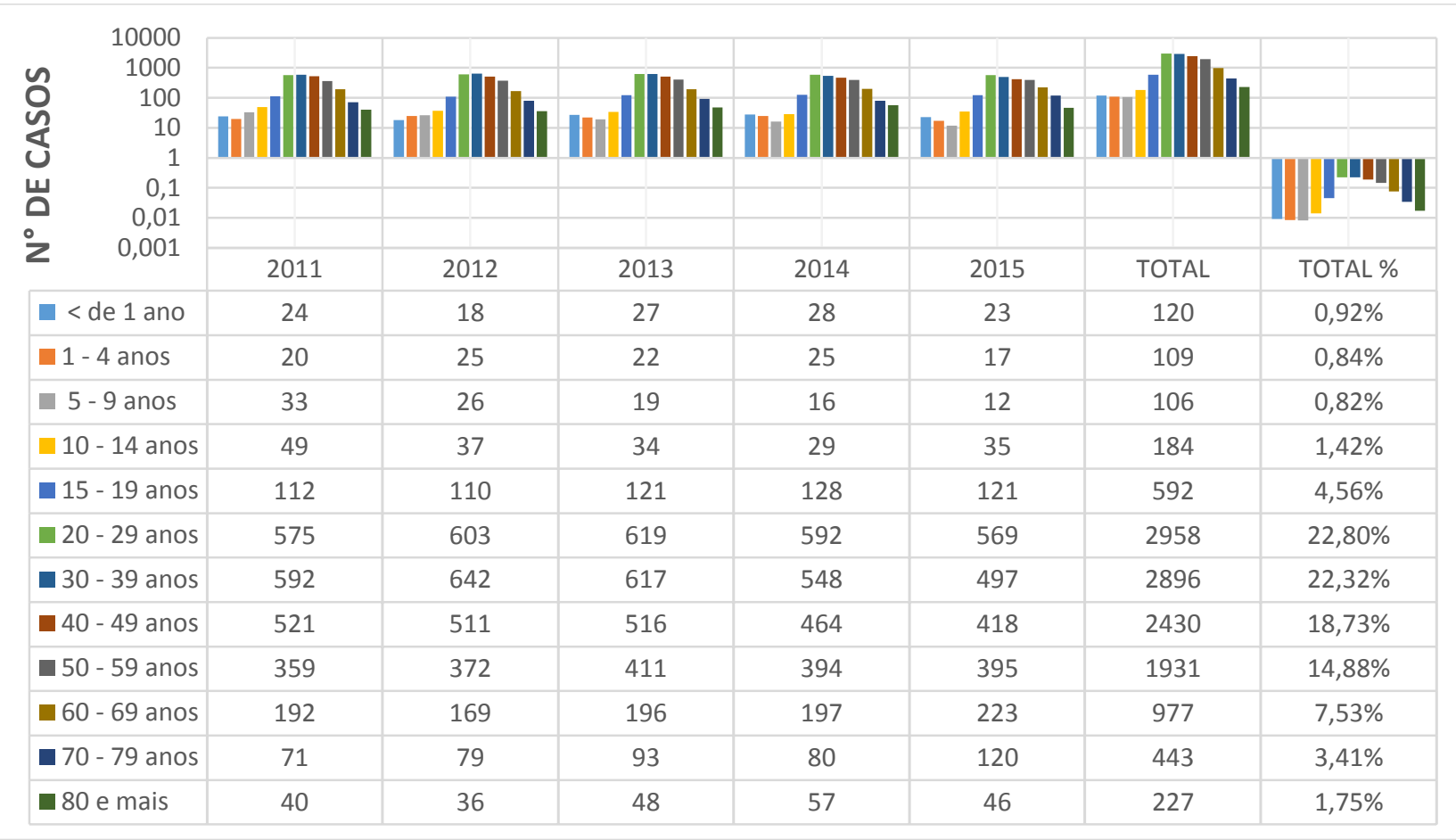

Fonte: SINAN 2011-2015, DATASUS. 
Em relação ao tipo de admissão, a maioria dessas admissões, 9.792 (75,48\%) ocorreram por caso NOVO, seguidos de reingresso pós abandono 1.591 (12,26\%) (Tabela 1). Com relação a distribuição etária, a faixa da população economicamente ativa (20-49 anos), foi a mais acometida com 5.854 ocorrências (45,12\%). Apesar de que em termos absolutos, a maioria dos casos ocorreu em indivíduos com idades entre 20-29 anos $(22,80 \%)$. Os idosos representaram $12,69 \%$ dos casos (Figura 1).

Conforme a institucionalização a maioria dos casos, $8.054(62,08 \%)$ ocorrem em indivíduos não institucionalizados, seguidos de ignorados/brancos 3.557 (27,73\%) e presidiários 963 (7,42\%) (Figura 2).

Figura 2 - Casos notificados de tuberculose no município de Recife/PE segundo institucionalização no período de 2011-2015.

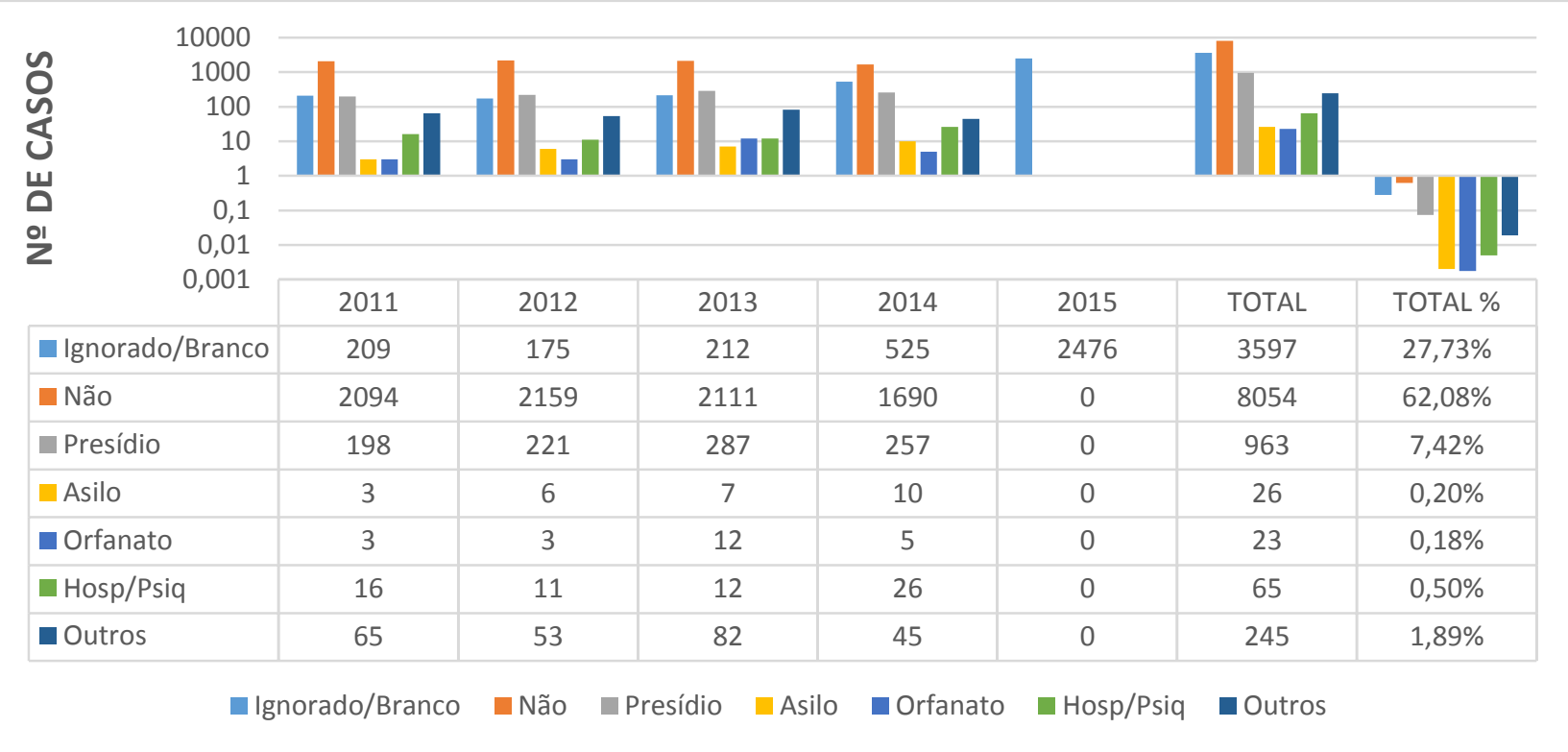

Fonte: SINAN 2011-2015, DATASUS.

No que se refere a baciloscopia, em $61,53 \%$ (7.982) dos casos foi realizada a baciloscopia, sendo 5.838 (45\%) POSITIVA e 2.144 (16,53\%) NEGATIVA. A baciloscopia não foi realizada em 4.897 (37,75\%) dos casos (Figura 3).

Figura 3 - Casos notificados de tuberculose no município de Recife/PE segundo realização de baciloscopia no período de 2011-2015.

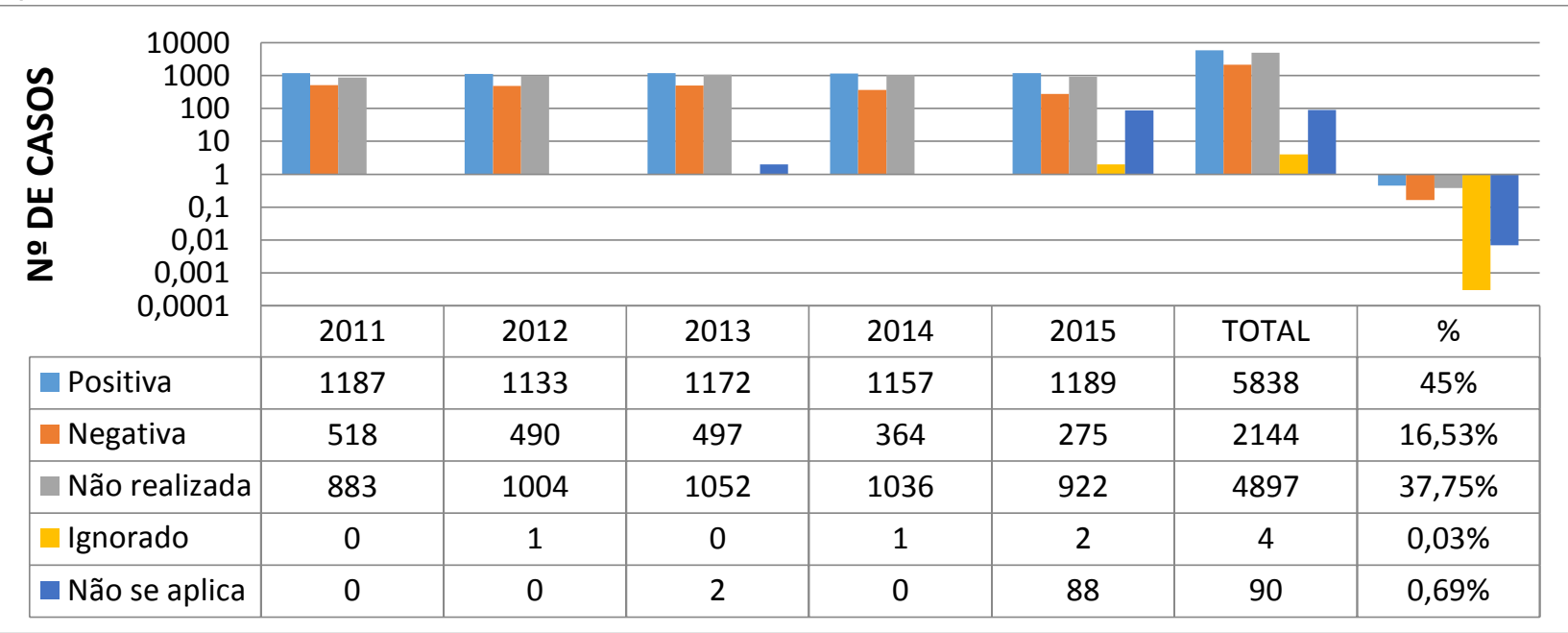

Fonte: SINAN 2011-2015, DATASUS. 
A sorologia para o HIV foi realizada em $57,76 \%$ (7.494) dos casos, onde teve o resultado negativo para $4.999(38,53 \%)$ e $2.495(19,23 \%)$ apresentaram sorologia positiva, porém um total de $4.330(33,38 \%)$ não realizaram a sorologia para o HIV (Figura 4).

Figura 4 - Casos notificados de tuberculose no município de Recife/PE segundo co-infecção TB/HIV no período de 2011-2015.

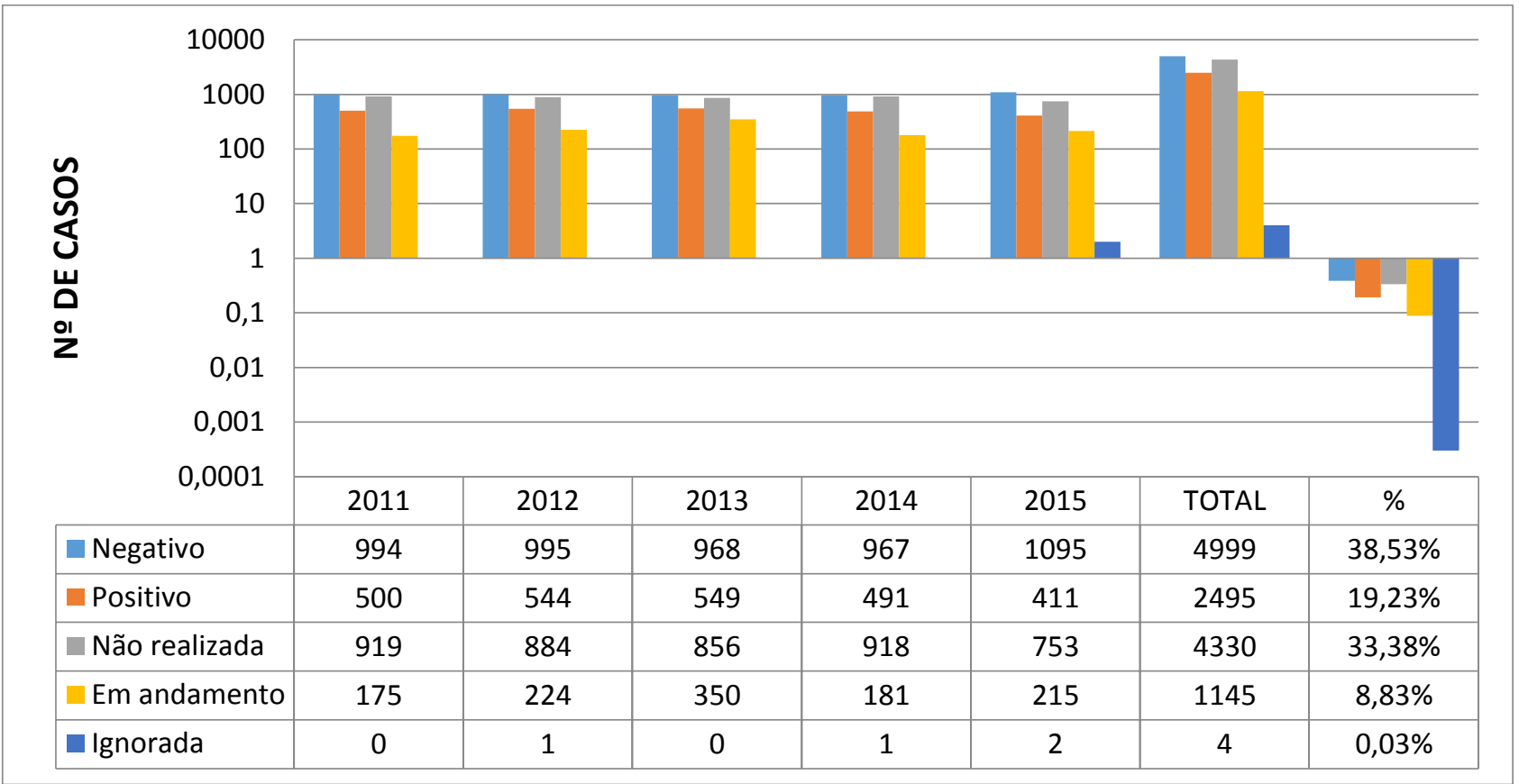

Fonte: SINAN 2011-2015, DATASUS.

Em relação a situação de encerramento dos casos de TB, a maioria evoluiu para a cura $6.013(46,35 \%)$, seguido do percentual de casos transferidos $2.172(16,74 \%)$, bem como 1.960 (15,11\%) casos de abandono do tratamento (Figura 5).

Figura 5 - Casos notificados de tuberculose no município de Recife/PE segundo situação de encerramento no período de 2011-2015.

\begin{tabular}{|c|c|c|c|c|c|c|c|}
\hline \multirow{3}{*}{ 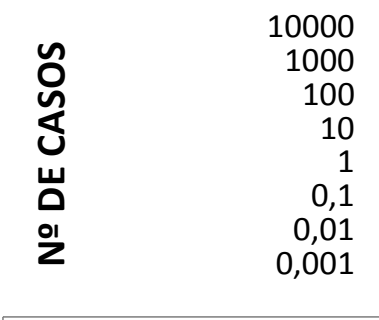 } & \multirow[b]{3}{*}{2011} & \multirow[b]{3}{*}{2012} & \multirow[b]{3}{*}{2013} & \multirow[b]{3}{*}{2014} & \multirow[b]{3}{*}{2015} & \multirow[b]{3}{*}{ TOTAL } & \multirow{3}{*}{$\%$} \\
\hline & & & & & & & \\
\hline & & & & & & & \\
\hline - Cura & 1314 & 1349 & 1397 & 1329 & 624 & 6013 & $46,35 \%$ \\
\hline Abandono & 444 & 467 & 472 & 372 & 205 & 1960 & $15,11 \%$ \\
\hline Óbito por TB & 116 & 172 & 185 & 174 & 161 & 808 & $6,23 \%$ \\
\hline Óbito por outras causas & 148 & 189 & 172 & 173 & 181 & 863 & $6,65 \%$ \\
\hline Transferência & 511 & 409 & 447 & 440 & 365 & 2172 & $16,74 \%$ \\
\hline TB Multiressistente & 14 & 17 & 9 & 14 & 5 & 59 & $0,45 \%$ \\
\hline Outros & 0 & 1 & 0 & 8 & 18 & 27 & $0,22 \%$ \\
\hline Ignorado/Branco & 38 & 27 & 41 & 48 & 917 & 1071 & $8,26 \%$ \\
\hline
\end{tabular}

Fonte: SINAN 2011-2015, DATASUS. 


\section{DISCUSSÃO}

O panorama apontado em Recife demonstra a incongruência de um município que, apesar de ser a capital do estado de Pernambuco, área com as melhores estruturas médico-sanitárias do estado, apresenta ainda dados exorbitantes de prevalência de tuberculose, sendo, portanto, prioritário para o combate da doença. Nota-se que os índices de casos notificados de tuberculose oscilaram pouco entre os anos em estudo, havendo uma discreta redução dos casos em 2014 e 2015.

A alta proporção da tuberculose em homens encontrada no nosso estudo também é predominante em vários estudos (OLIVEIRA JHS, et al., 2015; COSTA AFA, et al., 2016). Segundo a OMS, a doença afeta mais homens do que mulheres em razão de fatores econômicos, culturais e sociais. Os homens são mais acometidos devido a uma maior exposição pela sua vida social e menor procura de serviços de saúde, possibilitando maior chance de infecção com o bacilo (OMS, 2011).

Outra tendência verificada foi a apresentação clínica da doença, sendo a forma pulmonar a mais predominante com 10.487 casos $(80,84 \%)$. Tal achado está em concordância com a estimativa do Ministério da Saúde em que a forma pulmonar representa $80 \%$ dos casos (BRASIL, 2014).

Com relação à forma de admissão, 75,48\% (9.792) das entradas foram de casos novos. De acordo com Castelo Filho A, et al. (2004) esse fato reconhece a predisposição para a resolução da doença já nos primeiros atendimentos, seja nos serviços de níveis primários ou nos mais especializados, sendo bem menos procurado 0 atendimento de casos antigos. No decorrer do período estudado, notou-se ainda que o número de reingressos após abandono foi de 12,26\% (1.591). As grandes consequências de não se dar continuidade ao tratamento são o embate negativo no manejo da doença e tendência de se ter mais casos de tuberculose multirresistente, sendo essa condição mais grave, tendo um protocolo terapêutico mais complexo. (XAVIER MI, BARRETO ML, 2007).

A maior proporção dos casos de tuberculose em Recife ocorreu na faixa etária de 20 a 39 anos $(45,12 \%)$, semelhante ao relatado por Costa AFA et al. (2016) que observou em seu estudo um predomínio da faixa etária entre 20 a 39 anos (44\%) e Oliveira Junior HS et al. (2015) no qual evidenciou uma frequência maior de tuberculose na faixa etária entre 20 a 40 anos, atribuindo este achado ao fato das pessoas nesta fase não cuidarem muito de sua saúde e terem uma vida desregrada. Em países desenvolvidos, a faixa etária acima de 50 anos é a mais acometida pela doença, por outro lado, nos países em desenvolvimento notamos que os mais vulneráveis se encontram em idade produtiva, afetando desta maneira a economia do país (ARAÚJO KMFA, et al., 2013).

Quando investigada a situação de institucionalização do enfermo, verifica-se que $8,30 \%$ eram institucionalizados, sendo o presídio $(7,42 \%)$ a forma mais comum. O estudo de Barbosa IR e Henrique GL (2014) possui resultado inferior no qual, apenas, 3,3\% eram carcerários.

Os casos de Tuberculose na população privada de liberdade atraem atenção visto que o presídio é um local bastante favorável para a disseminação da tuberculose, devido a condições desfavoráveis, como aglomerações e a pouca ventilação (BARBOSA IR, HENRIQUE GL, 2014).

A baciloscopia, por ser um exame mais acessível, rápido e por ter um custo inferior aos demais, é um dos exames mais utilizado para ajudar no diagnóstico da tuberculose, entretanto o mesmo possui uma sensibilidade menor em relação a outros exames, como por exemplo a cultura do escarro. Neste estudo, a frequência em que o exame foi realizado $(61,53 \%)$ se mostrou inferior ao relatado por Coelho DMM et al (2010) em Teresina-PI com 82,11\% de realização deste exame.

Nesse estudo, chama atenção o alto percentual de casos em que não foi realizada a cultura de escarro $(37,75 \%)$, sendo que essa técnica é mais sensível que a baciloscopia e, também, apresenta a vantagem de permitir a recuperação da microbactéria para a identificação e testes de sensibilidade. Apesar de tecnicamente simples, a coleta, o armazenamento e o transporte das amostras de escarro devem ser feitas de maneira adequada, já que falhas no processo podem aumentar as taxas de contaminação da cultura e diminuir a sensibilidade da mesma (MACIEL ELN, et al., 2009). 
Quanto à análise de sorologia para HIV, foi efetuado em $57,76 \%$ dos casos, apresentando-se abaixo do definido pela meta do PNCT que é a realização do teste anti-HIV para $100 \%$ dos adultos com TB. A baixa porcentagem na solicitação de exames anti-HIV é o retrato da falta de conscientização dos profissionais de saúde a respeito da relevância da coinfecção TB/HIV (OMS, 2011).

Em relação a situação de encerramento dos casos de TB, a maioria evoluiu para a cura 6.013 (46,35\%), porém essa taxa detectada encontra-se abaixo do preconizado pelo Programa Nacional de Controle da Tuberculose (PNCT), que é atingir $85 \%$ de cura (BRASIL, 2011).

Mesmo com a descentralização das ações e a aproximação do doente aos serviços de saúde, a adesão ao tratamento da TB, ainda, configura como um dos principais obstáculos encontrados para a cura da doença. A não adesão culmina com o abandono, contribui para o insucesso terapêutico e proliferação da infecção (ALVES RS, et al., 2012). Neste estudo, foi identificado que a taxa de abandono nesse período foi de 1.960 casos $(15,11 \%)$. Apesar da limitação dos dados em consequência dos casos transferidos $(16,74 \%)$, os resultados apontam que as variáveis de encerramento do tratamento de TB no município são preocupantes, contribuindo para a manutenção das elevadas taxas de morbimortalidade.

\section{CONCLUSÃO}

Com a realização desta pesquisa, foi possível observar que a população masculina e de faixa etária economicamente ativa foi mais acometida pela tuberculose, apesar da maioria dos casos evoluir para a cura ainda não alcançamos a taxa de cura preconizada pelo PNCT. O desafio em se combater a doença ainda é muito grande, conhecer o perfil da população acometida possibilita a implantação de estratégias voltadas para a população mais vulnerável. Assim, o controle da tuberculose depende de melhoria dos fatores relacionados aos serviços de saúde: sistema de informação confiável, ampliação dos atendimentos descentralizados que proporcionam a implementação do tratamento supervisionado, capacitação dos profissionais, fortalecimento do trabalho em equipe e otimização de fluxograma de referência e contra referência.

\section{REFERÊNCIAS}

1. ALVES RS, et al. Abandono do tratamento da tuberculose e integralidade da atenção na estratégia saúde da família. Texto Contexto-Enfermagem, Florianópolis, 2012; 21(3): 650-657.

2. ARAÚJO KMFA, et al. Evolução da distribuição espacial dos casos novos de tuberculose no município de Patos (PB), 2001-2010. Cadernos Saúde Coletiva, 2013; 21(3): 296-302.

3. ARAÚJO SRL, et al. Perfil epidemiológico da tuberculose pulmonar na cidade de Natal-RN. Journal of Infection Control, 2015; 4(1): 16-19.

4. BARBOSA IR, HENRIQUE GL. Caracterização dos casos de tuberculose em um município prioritário no estado do Rio Grande do Norte. Revista de APS, 2014; 17(1): 24-31.

5. BOMBARDA S, et al. Recomendações para o diagnóstico e tratamento das micobacterioses não tuberculosas no Estado de São Paulo. Divisão de Tuberculose, Centro de Vigilância Epidemiológica Alexandre Vranjak, Secretaria de Estado da Saúde de São Paulo, Brasil. 2014; 6(3).

6. Brasil. Ministério da Saúde. Secretaria de vigilância em saúde. Programa Nacional de Controle da Tuberculose. Manual de recomendações para o controle da Tuberculose no Brasil. Brasília: 2011.

7. BRASIL. Ministério da Saúde. Secretaria de Vigilância em Saúde. Boletim Epidemiológico. 2015; 46(9).

8. BRASIL. Ministério da Saúde. Secretaria de Vigilância em Saúde. Departamento de Vigilância das Doenças Transmissíveis. Plano nacional pelo fim da tuberculose / Ministério da Saúde, Secretaria de Vigilância em Saúde, Departamento de Vigilância das Doenças Transmissíveis. Brasília: Ministério da Saúde, 2017.

9. CASTELO FILHO A, et al. II Consenso Brasileiro de Tuberculose: Diretrizes Brasileiras para Tuberculose 2004. Jornal Brasileiro de Pneumologia, 2004; 30(Supl 1): S1-S56.

10. CHAVES J, et al. Perfil dos pacientes com tuberculose que foram atendidos em uma unidade de referência do município de Santa Cruz do Sul-RS no período de 2009 a 2013. Journal of Health \& Biological Sciences, 2017; 5(1): 31-36.

11. COELHO DMM, et al. Perfil epidemiológico da tuberculose no Município de Teresina-PI, no período de 1999 a 2005. Epidemiologia e Serviços de Saúde. 2010; 19(1): 34-43. 
12. COSTA AFA, et al. Tuberculosis' epidemiological profile: historic série. Journal of Nursing UFPE on line, 2016;10(6):1957-1962.

13. FREITAS VMTM, et al. Perfil clínico-epidemiológico de pacientes portadores de tuberculose atendidos em uma unidade municipal de saúde de Belém, Estado do Pará, Brasil. Revista Pan-Amazônica de Saúde, 2016; 7(2): 4550.

14. LIMA SS, et al. Análise espacial da tuberculose em Belém, estado do Pará, Brasil. Revista Pan-Amazônica de Saúde, 2017; 8(2): 55-63.

15. MACIEL ELN, et al. Associação entre coleta de escarro guiada e taxas de contaminação de culturas para o diagnóstico de TB pulmonar. Jornal Brasileiro de Pneumologia. 2009; 35(5): 460-463.

16. OLIVEIRA GM, PETRONI TF. Avaliação de indicadores epidemiológicos da tuberculose no Brasil. Revista Saúde UniToledo, 2017; 1(1): 134-146.

17. OLIVEIRA JUNIOR HS, et al. Prevalência de Casos de Tuberculose Durante os Anos de 2002 a 2012 , no Município de Palmas-Paraná, Brasil. Revista de Saúde Pública de Santa Catarina, 2015; 8(1): 43-57.

18. OMS. World Health Organization. Global Tuberculosis Control. TB prevalence surveys: a handbook. Geneva: World Health Organization, 2011.

19. PEREIRA VLT, et al. A incidência de tuberculose no Vale do Ribeira. Revista Gestão em Foco, 2017; 9: 90-102.

20. XAVIER MIM, BARRETO ML. Tuberculose na cidade de Salvador, Bahia, Brasil: o perfil na década de 1990. Caderno de Saúde Pública, 2007; 23(2): 445-453. 